\title{
Stability and efficacy of sunscreens containing inorganic filters and quercetin
}

\author{
Estabilidade e eficácia de fotoprotetores contendo filtros inorgânicos e quercetina
}

\section{Deborah de Oliveira Nishikawa, Daniela D'Almeida Peres, Camila Areias de Oliveira, Vânia Rodrigues Leite e Silva, Telma Mary Kaneko, Maria Valéria Robles Velasco \& André Rolim Baby}

School of Pharmaceutical Sciences, University of São Paulo. São Paulo, SP, Brazil

Email: daniela.peres@usp.br

Fontes de Financiamento: FAPESP e CNPq

\begin{abstract}
Exposure to the sun can have health benefits, however, excess exposure can cause skin damage, such as skin cancer. Photoprotection can be used as a preventive method against the harmful effects of the ultraviolet (UV) radiation and Brazilian biodiversity is a fertile field for research in this area. Therefore, the aim of this study was the development of sunscreens containing quercetin (bioactive compound) and inorganic filters (titanium dioxide and zinc oxide) with further characterization of the formulae and evaluation of stability. Formulations containing the bioactive compound, isolated or in association with the inorganic filters, presented: $\mathrm{pH}$ values biocompatible with the skin; apparent viscosity between 10550 and $23600 \mathrm{cP}$; sun protection factor (SPF) between 2.1 and 22.5; and broad spectrum protection, with critical wavelengths above $379 \mathrm{~nm}$. It was found that the association of quercetin with inorganic filters was not adequate due to the negative interactions that occurred between the bioactive compound and the metals, which were only identified during the stability study. However, considering the in vitro estimated efficacy of the isolated flavonoid, its use can still be explored as an alternative substitute to classic UV filters.
\end{abstract}

Keywords: Photoprotection; Quercetin; Stability; Titanium dioxide, Zinc oxide.

\begin{abstract}
Resumo
A exposição ao sol traz benefícios à saúde, no entanto, o excesso pode ocasionar danos cutâneos dentre os quais se destacam as neoplasias. A fotoproteção é um método para a prevenção dos efeitos danosos da radiação ultravioleta (UV) e a biodiversidade Brasileira é campo fértil para pesquisas nesta área. Dessa forma, os objetivos deste estudo envolveram o desenvolvimento de formulações fotoprotetoras contendo quercetina (composto bioativo) e filtros solares físicos (dióxido de titânio e óxido de zinco), com posterior caracterização das formulações e avaliação da sua estabilidade. As formulações contendo o composto bioativo, isolado ou em associação com os filtros físicos, possuíram valores de $\mathrm{pH}$ biocompatíveis com a pele, intervalo de viscosidade aparente entre 10550 e 23600 cP; fator de proteção solar (FPS) estimado entre 2.1 e 22.5; e amplo espectro de proteção, com comprimentos de onda crítico acima de $379 \mathrm{~nm}$. Constatou-se que não foi adequado utilizar a quercetina associada aos filtros solares físicos devido às interações negativas que ocorreram entre o composto e os metais, somente identificadas ao longo do estudo de estabilidade. No entanto, em função da eficácia estimada in vitro apresentada pelo flavonoide, seu uso ainda pode ser explorado como substituto alternativo aos filtros solares clássicos.
\end{abstract}

Palavras-chave: Dióxido de titânio; Estabilidade; Fotoproteção; Óxido de zinco, Quercetina. 


\section{Introduction}

The sunlight that reaches the Earth's surface is composed of electromagnetic radiation, divided in three intervals according to its wavelength: ultraviolet (UV) (100-400 $\mathrm{nm}$ ), visible (Vis) $(400-800 \mathrm{~nm})$, and infrared (IR) (above $800 \mathrm{~nm})^{[1,2]}$.

Photoprotection is a method for preventing the harmful effects of UV radiation and involves the use of sunscreens containing inorganic (physical) and organic (chemical) filters ${ }^{[3,4,5]}$.

Sunscreens contribute to the reduction of skin cancer incidence and photoaging signs. However, nowadays the history of adverse events caused by the topical use of UV chemical filters has led the market to develop formulations containing low concentrations of chemical filters, whilst maintaining the broad spectrum protection against UVA and UVB radiation ${ }^{[6,7]}$

In spite of the different classes of UV filters and natural substances available, the market did not present photoprotective formulations composed of an association of UV filters and isolated flavonoids. In accordance with this prospect, the present study proposed the development of bioactive photoprotective formulations, without chemical filters, and aims to evaluate the quercetin flavonoid as a UV filter. The stability of the formulations containing quercetin, associated or not with the inorganic filters titanium dioxide and zinc oxide, was also evaluated by this study.

\section{Material and Methods}

\section{Formulations}

Quercetin and inorganic filters (titanium dioxide and zinc oxide) were incorporated in an oil-in-water $(\mathrm{O} / \mathrm{W})$ emulsion, according to the qualitative and quantitative $(\% \mathrm{w} / \mathrm{w})$ composition described in Table 1.

\section{Formulations characterization pH value determination}

The $\mathrm{pH}$ value was determined by $\mathrm{pHmeter}$ Quimis ${ }^{\circledR}$, using a $10 \% \mathrm{w} / \mathrm{V}$ dispersion of the formulations. The readings were performed at room temperature $\left(22.0 \pm 2.0^{\circ} \mathrm{C}\right)^{[8,9]}$.

\section{Apparent viscosity (cP)}

Apparent viscosity (cP) was determined by the ViscoStar $^{\circledR}$ viscosimeter. Samples of approximately 10.0 $\mathrm{g}$ of each formulation were transferred to the viscosimeter compartment of reduced samples, and the analyses were performed at room temperature $(22.0 \pm$ $2.0^{\circ} \mathrm{C}$ ), at $30 \mathrm{rpm}$, using the TR9 spindle. Measurements were made after three minutes of rotation.

\section{Introdução}

A luz solar incidente sobre a superfície terrestre é composta por radiação eletromagnética, dividida em três intervalos majoritários de acordo com seu comprimento de onda: ultravioleta (UV) (100-400 nm), visível (Vis) (400-800 nm), e infravermelho (IV) (acima de $800 \mathrm{~nm})^{[1,2]}$.

A fotoproteção é um método para a prevenção dos efeitos danosos da radiação UV, abordagem realizada por meio do uso de fotoprotetores contendo filtros inorgânicos (físicos) e orgânicos (químicos) ${ }^{[3,4,5]}$.

Os protetores solares atuam como adjuvantes na redução da incidência de câncer de pele e dos sinais de envelhecimento, porém, atualmente, devido ao histórico dos eventos adversos ocasionados pelo uso tópico de grandes concentrações de filtros químicos, a tendência no mercado de fotoprotetores é o desenvolvimento de formulações contendo reduzida concentração de filtros químicos, mas com manutenção da ampla proteção frente às radiações UVA e UVB ${ }^{[6,7]}$.

Apesar da disponibilidade de diferentes classes de filtros solares e substâncias naturais isoladas, o mercado não dispõe de formulações fotoprotetoras possuindo associações de filtros solares com flavonoides isolados. Em concordância com este panorama, o presente trabalho envolveu $o$ desenvolvimento de formulações fotoprotetoras bioativas ausentes de filtros solares químicos, com o objetivo de avaliar o uso do flavonoide quercetina como filtro solar. Este trabalho avaliou, também, a estabilidade das formulações contendo quercetina, associada ou não aos filtros físicos dióxido de titânio e óxido de zinco.

\section{Materiais e Métodos}

\section{Formulações}

A quercetina e os filtros físicos (dióxido de titânio e óxido de zinco) foram incorporados em emulsão óleoem-água $(\mathrm{O} / \mathrm{A})$ de acordo com a composição qualitativa e quantitativa $(\% \mathrm{p} / \mathrm{p})$ descrita na Tabela 1 .

\section{Caracterização das Formulações \\ Determinação do valor de $\mathbf{p H}$}

$\mathrm{O}$ valor de $\mathrm{pH}$ foi determinado pela dispersão a $10 \% \mathrm{p} / \mathrm{v}$ das preparações analisadas à temperatura ambiente $(22.0$ $\pm 2.0^{\circ} \mathrm{C}$ ), em peagômetro Quimis ${ }^{\mathbb{B}[8,9]}$.

\section{Viscosidade aparente (cP)}

A viscosidade aparente $(\mathrm{cP})$ foi determinada em viscosímetro ViscoStar ${ }^{\circledR}$. Alíquotas de $10.0 \mathrm{~g}$ das amostras foram transferidas para o compartimento de amostra reduzida. As medições foram realizadas à temperatura ambiente $\left(22.0 \pm 2.0{ }^{\circ} \mathrm{C}\right)$, utilizando-se agulha TR9 e velocidade de rotação de $30 \mathrm{rpm}$. O registro do valor de viscosidade aparente foi realizado após 3 minutos de rotação. 
Table 1 - Formulations qualitative and quantitative $(\% \mathrm{w} / \mathrm{w})$ composition

Tabela 1 - Composição qualitativa e quantitativa $(\%$ p/p) das formulações

\begin{tabular}{|c|c|c|c|c|c|c|}
\hline \multirow{2}{*}{$\begin{array}{l}\text { Composition } \\
\text { Composição }\end{array}$} & \multicolumn{6}{|c|}{$\begin{array}{c}\text { Proportion }(\% \mathrm{w} / \mathbf{w}) \\
\text { Proporção }(\% \mathrm{p} / \mathrm{p})\end{array}$} \\
\hline & F1 & F2 & F3 & F4 & F5 & F6 \\
\hline $\begin{array}{c}\text { Cetearyl Alcohol (and) Glyceryl Stearate } \\
\text { (and) PEG-2 Stearate (and) Stearic Acid } \\
\text { (and) Ceteth-10 (and) Polysorbate } 60 \text { (and) } \\
\text { Theobroma grandiflorum seed butter (and) } \\
\text { Helianthus annuus seed oil (and) Cetyl } \\
\text { Palmitate }\end{array}$ & 10.0 & 10.0 & 10.0 & 10.0 & 10.0 & 10.0 \\
\hline BHT & 0.1 & 0.1 & 0.1 & 0.1 & 0.1 & 0.1 \\
\hline Glycerin & 4.0 & 4.0 & 4.0 & 4.0 & 4.0 & 4.0 \\
\hline Sorbitol & 4.0 & 4.0 & 4.0 & 4.0 & 4.0 & 4.0 \\
\hline Disodium EDTA & 0.5 & 0.5 & 0.5 & 0.5 & 0.5 & 0.5 \\
\hline $\begin{array}{c}\text { Cyclopentasiloxane (and) Dimethicone } \\
\text { Crosspolymer }\end{array}$ & 2.0 & 2.0 & 2.0 & 2.0 & 2.0 & 2.0 \\
\hline $\begin{array}{c}\text { Mineral Oil (and) Lanolin Alcohol (and) } \\
\text { Oleyl Alcohol }\end{array}$ & 2.0 & 2.0 & 2.0 & 2.0 & 2.0 & 2.0 \\
\hline Cyclopentasiloxane & 2.0 & 2.0 & 2.0 & 2.0 & 2.0 & 2.0 \\
\hline Imidazolidinyl Ureia & 0.5 & 0.5 & 0.5 & 0.5 & 0.5 & 0.5 \\
\hline $\begin{array}{c}\text { Butilparaben (and) Ethylparaben (and) } \\
\text { Methylparaben (and) Phenoxyethanol (and) } \\
\text { Propylparaben }\end{array}$ & 0.5 & 0.5 & 0.5 & 0.5 & 0.5 & 0.5 \\
\hline Quercetin & - & 2.5 & - & 2.5 & 2.5 & 2.5 \\
\hline Zinc oxide & - & 5.0 & 5.0 & - & 5.0 & - \\
\hline Titanium dioxide & - & 5.0 & 5.0 & 5.0 & - & - \\
\hline Aqua qsp** & 100.0 & 100.0 & 100.0 & 100.0 & 100.0 & 100.0 \\
\hline
\end{tabular}

Legend:(-): raw material not incorporated.

* Qualitative composition described according to INCI (International Nomenclature of Cosmetic Ingredients)

Legenda:(-): matéria-prima não adicionada.

* Composição qualitativa descrita segundo nomenclatura INCI (International Nomenclature of Cosmetic Ingredients)

\section{In vitro photoprotective efficacy}

The in vitro photoprotective efficacy of the formulations was measured by spectral transmittance using an integrating sphere (Labsphere ${ }^{\circledR}$ UV-2000S). The following parameters were obtained: estimated sun protection factor (SPF) and critical wavelength $\left(\lambda_{C}\right)$.

Commercial plates of polymethyl methacrylate (PMMA HD6, Helioplate ${ }^{\mathbb{R}}$ ) were used as a synthetic substrate. Aliquots of $50.0 \mathrm{mg}$ of the samples were uniformly applied on the substrate, as a thin layer, in circular movements, over the plate's surface.

The spectrophotometric absorbance values were registered for the wavelength interval of 250 to $450 \mathrm{~nm}$, with a rate progression of $1 \mathrm{~nm}$. The plates were

\section{Eficácia fotoprotetora in vitro}

Determinou-se a eficácia in vitro das preparações em espectrofotômetro de refletância difusa com esfera de integração (Labsphere ${ }^{\circledR}$ UV2000-S) por meio da obtenção dos parâmetros a seguir: fator de proteção solar estimado (FPS) e comprimento de onda crítico $\left(\lambda_{\mathrm{C}}\right)$. Utilizaram-se, como substrato sintético, placas comerciais de polimetilmetacrilato (PMMA HD6), Helioplate $^{\circledR}$. Alíquotas de $50.0 \mathrm{mg}$ das amostras foram aplicadas uniformemente sobre o substrato, na forma de filme fino, em movimentos circulares sobre a superfície da placa. Os registros dos valores espectrofotométricos da transmitância foram realizados em intervalo de comprimento de onda entre 
prepared in replicates of two and nine different points per plate were measured for each sample ${ }^{[10,11,12,13]}$. The samples' estimated functionality values were calculated by the equipment software (Labsphere ${ }^{\circledR}$ UV-2000 Application Version: 1.1.1.0)

\section{Stability test}

Samples of approximately $20.0 \mathrm{~g}$ were stored in polypropylene wide-mouth bottles, occupying $2 / 3$ of the total declared volume of the bottle, for evaluation of possible gas exchanges. Samples were stored under the following conditions: (i) $40.0^{\circ} \mathrm{C} \pm 2.0^{\circ} \mathrm{C}$ (incubator), (ii) $22.0^{\circ} \mathrm{C} \pm 2.0^{\circ} \mathrm{C}$ (room temperature), and (iii) $5.0^{\circ} \mathrm{C} \pm$ $2.0^{\circ} \mathrm{C}$ (refrigerator). Organoleptic characteristics, $\mathrm{pH}$ value, apparent viscosity and in vitro photoprotective efficacy were evaluated at the $1^{\text {st }}, 30^{\text {th }}, 60^{\text {th }}, 90^{\text {th }}$ and $120^{\text {th }}$ day ${ }^{[14]}$.

\section{Results}

Formulations were stable after the preparation (initial condition), according to the data presented in Table 2 . Homogeneous emulsions were obtained with color varying between white and yellow, and $\mathrm{pH}$ and apparent viscosity values were compatible with the skin and the proposed use of the product. The SPF values varied from $1.1 \pm 0.0$ (base emulsion, represented by the formulation F1) and $22.5 \pm 7.2$ (formulation F2, containing $2.5 \% \mathrm{w} / \mathrm{w}$ of quercetin, $5.0 \% \mathrm{w} / \mathrm{w}$ of titanium dioxide and $5.0 \% \mathrm{w} / \mathrm{w}$ of zinc oxide). All formulations presented high values of critical wavelength $\left(\lambda_{c}\right)$, above $379 \mathrm{~nm}$, illustrating the formulations absorption on the UVA radiation spectrum.
250 e $450 \mathrm{~nm}$, na taxa de progressão de $1 \mathrm{~nm}$. Leituras em duplicata foram empregadas com repetições de nove leituras por réplica ${ }^{[10,11,12,13]}$. Os valores de funcionalidade estimada das amostras foram calculados pelo software do equipamento (Labsphere ${ }^{\circledR}$ UV-2000 Application Versão: 1.1.1.0).

\section{Teste de estabilidade}

Amostras de, aproximadamente, $20.0 \mathrm{~g}$ foram acondicionadas em potes de polipropileno, boca larga, ocupando cerca de $2 / 3$ do volume total declarado do frasco para avaliação de possíveis trocas gasosas. As amostras foram submetidas às seguintes condições: (i) $40.0^{\circ} \mathrm{C} \pm 2.0^{\circ} \mathrm{C}$ (estufa), (ii) a temperatura ambiente a $22.0^{\circ} \mathrm{C} \pm 2.0^{\circ} \mathrm{C}$ e (iii) $5.0^{\circ} \mathrm{C} \pm 2.0^{\circ} \mathrm{C}$ (refrigerador). As características organolépticas (odor, cor e aspecto), valor de $\mathrm{pH}$, viscosidade aparente e eficácia fotoprotetora in vitro foram avaliados no $1^{\circ}, 30^{\circ}, 60^{\circ}, 90^{\circ}$ e $120^{\circ}$ dias ${ }^{[14]}$.

\section{Resultados}

As preparações apresentaram-se estáveis após a etapa de manipulação (condição inicial), conforme parâmetros apresentados na Tabela 2. Foram obtidas emulsões homogêneas, com coloração variando de branco a amarelado, e valores de $\mathrm{pH}$ e viscosidade aparente compatíveis com a pele e o uso proposto do produto. Os valores de FPS variaram entre $1.1 \pm 0.0$ (emulsão base, representada pela formulação F1) e $22.5 \pm 7.2$ (formulação $\mathrm{F} 2$, contendo $2.5 \% \mathrm{p} / \mathrm{p}$ de quercetina, $5.0 \% \mathrm{p} / \mathrm{p}$ de dióxido de titânio e $5.0 \% \mathrm{p} / \mathrm{p}$ de óxido de zinco). Todas as formulações apresentaram valores de comprimento de onda crítico $\left(\lambda_{c}\right)$ elevados, acima de $379 \mathrm{~nm}$, caracterizando o perfil de absorção das formulações no espectro de radiação UVA.

Table 2 - Formulations physical and physical-chemical characterization after preparation (initial condition) Tabela 2 - Caracterização física e físico-química das formulações após a manipulação (condição inicial)

\begin{tabular}{|c|c|c|c|c|c|c|}
\hline \multirow{2}{*}{$\begin{array}{c}\text { Evaluated } \\
\text { parameter } \\
\text { Parâmetro avaliado }\end{array}$} & \multicolumn{6}{|c|}{$\begin{array}{c}\text { Formulations } \\
\text { Formulações }\end{array}$} \\
\hline & F1 & F2 & F3 & F4 & F5 & F6 \\
\hline $\begin{array}{l}\text { Aspect } \\
\text { Aspecto }\end{array}$ & $\begin{array}{c}\text { White } \\
\text { emulsion } \\
\text { Emulsão } \\
\text { Branca }\end{array}$ & $\begin{array}{c}\text { Yellow } \\
\text { emulsion } \\
\text { Emulsão } \\
\text { amarela }\end{array}$ & $\begin{array}{c}\text { White } \\
\text { emulsion } \\
\text { Emulsão } \\
\text { branca }\end{array}$ & $\begin{array}{c}\text { Yellow } \\
\text { emulsion } \\
\text { Emulsão } \\
\text { amarela }\end{array}$ & $\begin{array}{c}\text { Yellow } \\
\text { emulsion } \\
\text { Emulsão } \\
\text { amarela }\end{array}$ & $\begin{array}{c}\text { Yellow } \\
\text { emulsion } \\
\text { Emulsão } \\
\text { amarela }\end{array}$ \\
\hline $\begin{array}{l}\text { SPF } \\
\text { FPS }\end{array}$ & $1.1 \pm 0.0$ & $22.5 \pm 7.2$ & $11.3 \pm 1.5$ & $12.3 \pm 2.1$ & $7.4 \pm 0.9$ & $2.1 \pm 0.2$ \\
\hline $\begin{array}{l}\lambda_{\mathrm{c}} \\
\lambda_{\mathrm{c}}\end{array}$ & 386 & 385 & 379 & 388 & 389 & 391 \\
\hline $\begin{array}{l}\text { pH value } \\
\text { Valor de } \mathrm{pH}\end{array}$ & 4.73 & 6.97 & 7.16 & 5.43 & 7.26 & 4.81 \\
\hline $\begin{array}{l}\text { Apparent viscosity } \\
\text { Viscosidade } \\
\text { aparente }\end{array}$ & 10550 & 12400 & 23600 & 23600 & 16000 & 20200 \\
\hline
\end{tabular}

Legend: SPF: Sun Protection Factor (média \pm desvio padrão) $\lambda \mathrm{c}$ : Critical wavelenght

Legenda: FPS: Fator de Proteção Solar (average \pm standard deviation) $\lambda c$ : Comprimento de onda crítico 
Formulation F1 did not present active compounds, since it was used during the stability test as a control for the base emulsion behavior evaluation. According to the data presented in Table 3, formulation F1 presented adequate stability, with maintenance of its homogeneous aspect and without alteration of its organoleptic characteristics. The $\mathrm{pH}$ value was stable, without variation above $15 \%$ when compared to the initial values obtained for all the storage conditions studied. The apparent viscosity in room $\left(22.0 \pm 2.0^{\circ} \mathrm{C}\right)$ and high temperatures $\left(40.0 \pm 2.0^{\circ} \mathrm{C}\right)$ storage showed an increasing tendency when compared to the initial condition. Although the apparent viscosity presented variations, this characteristic did not influence the product application on the skin. Under low temperature storage $\left(5.0 \pm 2.0^{\circ} \mathrm{C}\right)$, apparent viscosity were stable, with a decreasing tendency in the $120^{\text {th }}$ day of analysis.
A formulação $F 1$ não possuiu compostos ativos incorporados, tendo sido utilizada, durante o período do teste de estabilidade, como parâmetro controle para avaliar o comportamento da emulsão base. De acordo com os dados da Tabela 3, verificou-se que a formulação F1 apresentou estabilidade adequada, com manutenção do seu aspecto homogêneo, sem alterações das características organolépticas (aspecto, cor e odor). $\mathrm{O}$ valor de $\mathrm{pH}$ se manteve estável, não ocorrendo variação superior a $15 \%$ em relação ao valor inicial em nenhuma das condições estudadas. $O$ valor de viscosidade aparente apresentou tendência de aumento em relação ao valor inicial nas condições de temperatura ambiente $\left(22.0 \pm 2.0^{\circ} \mathrm{C}\right)$ e temperatura elevada $\left(40.0 \pm 2.0^{\circ} \mathrm{C}\right)$. Apesar do valor da viscosidade aparente ter apreciado variações, esta característica não influenciou no perfil de aplicação do produto sobre a pele. Em condição de baixa temperatura, a viscosidade aparente se manteve estável, com tendência de queda no $120^{\circ}$ dia de análise.

Table 3 - Stability evaluation of formulations F1, F3 e F6: pH value, apparent viscosity (cP) and in vitro functional characterization.

Tabela 3 - Avaliação da estabilidade das formulações F1, F3 e F6: valor de pH, viscosidade aparente (cP) e caracterização funcional in vitro.

\begin{tabular}{|c|c|c|c|c|c|c|c|c|c|c|c|c|c|}
\hline \multirow{3}{*}{$\begin{array}{c}\text { Formulation } \\
\text { Formulação }\end{array}$} & \multirow{3}{*}{$\begin{array}{c}\text { Evaluated } \\
\text { parameter } \\
\text { Parâmetro } \\
\text { avaliado }\end{array}$} & \multicolumn{12}{|c|}{$\begin{array}{c}\text { Storage condition } \\
\text { Condição de armazenamento }\end{array}$} \\
\hline & & \multicolumn{4}{|c|}{$\begin{array}{l}\text { Room temperature } \\
\text { Temperatura ambiente } \\
\left(\mathbf{2 2 . 0} \pm \mathbf{2 . 0}{ }^{\circ} \mathbf{C}\right)\end{array}$} & \multicolumn{4}{|c|}{$\begin{array}{c}\text { Low temperature } \\
\text { Temperatura baixa } \\
\left(\mathbf{5 . 0} \pm \mathbf{2 . 0}{ }^{\circ} \mathbf{C}\right) \\
\end{array}$} & \multicolumn{4}{|c|}{$\begin{array}{c}\text { High temperature } \\
\text { Temperatura elevada } \\
\left(\mathbf{4 0 . 0} \pm \mathbf{2 . 0}{ }^{\circ} \mathbf{C}\right)\end{array}$} \\
\hline & & T30 & T60 & T90 & T120 & T30 & T60 & T90 & T120 & T30 & T60 & T90 & T120 \\
\hline \multirow{4}{*}{ F1 } & $\begin{array}{l}\text { SPF } \\
\text { FPS }\end{array}$ & $1.0 \pm 0.0$ & $\begin{array}{c}1.1 \pm \\
0.0\end{array}$ & $\begin{array}{c}1.1 \pm \\
0.0 \\
\end{array}$ & $\begin{array}{c}1.1 \pm \\
0.0\end{array}$ & $\begin{array}{c}1.1 \pm \\
0.0 \\
\end{array}$ & $\begin{array}{c}1.1 \pm \\
0.0 \\
\end{array}$ & $\begin{array}{c}1.1 \pm \\
0.0 \\
\end{array}$ & $\begin{array}{c}1.1 \pm \\
0.0\end{array}$ & $1.1 \pm 0.0$ & $\begin{array}{c}1.1 \pm \\
0.0\end{array}$ & $\begin{array}{c}1.1 \pm \\
0.0\end{array}$ & $\begin{array}{c}1.1 \pm \\
0.1\end{array}$ \\
\hline & $\lambda_{c}$ & 359 & 373 & 362 & 370 & 375 & 378 & 377 & 378 & 376 & 376 & 371 & 377 \\
\hline & $\begin{array}{l}\text { pH value } \\
\text { Valor de pH }\end{array}$ & 4.48 & 4.90 & 4.83 & 4.87 & 4.54 & 4.84 & 4.57 & 4.87 & 4.63 & 4.56 & 4.74 & 4.84 \\
\hline & $\begin{array}{l}\text { Apparent } \\
\text { viscosity } \\
\text { Viscosidade } \\
\text { aparente }\end{array}$ & 17300 & 21100 & 19800 & 21650 & 10700 & 10400 & 10250 & 8600 & 21100 & 24050 & $\begin{array}{c}2515 \\
0\end{array}$ & 25950 \\
\hline \multirow{4}{*}{ F3 } & $\begin{array}{l}\text { SPF } \\
\text { FPS }\end{array}$ & $11.1 \pm 3.0$ & $\begin{array}{c}11.9 \pm 2 . \\
1\end{array}$ & $\begin{array}{c}12.5 \pm 2 . \\
4\end{array}$ & $\begin{array}{c}11.9 \pm 2 . \\
3\end{array}$ & $\begin{array}{c}12.3 \pm 2 \\
5\end{array}$ & $\begin{array}{c}12.2 \pm 3 \\
1\end{array}$ & $\begin{array}{c}12.7 \pm 1 \\
7\end{array}$ & $\begin{array}{c}12.8 \pm 1 \\
7\end{array}$ & $12.1 \pm 2.0$ & $\begin{array}{c}12.7 \pm 2 \\
8\end{array}$ & $\begin{array}{r}11.8 \\
\pm 1.6 \\
\end{array}$ & $\begin{array}{c}12.6 \pm 2 . \\
2\end{array}$ \\
\hline & $\lambda_{c}$ & 376 & 377 & 378 & 378 & 378 & 377 & 379 & 378 & 378 & 378 & 379 & 378 \\
\hline & $\begin{array}{l}\text { pH value } \\
\text { Valor de } \mathrm{pH}\end{array}$ & 7.64 & 6.95 & 7.13 & 7.81 & 7.45 & 7.38 & 7.17 & 7.76 & 7.62 & 7.55 & 7.23 & 7.75 \\
\hline & $\begin{array}{c}\text { Apparent } \\
\text { viscosity } \\
\text { Viscosidade } \\
\text { aparente }\end{array}$ & 32300 & 35350 & 34800 & 32600 & 16100 & 17350 & 16700 & 16200 & 38350 & 38100 & $\begin{array}{c}4015 \\
0\end{array}$ & 39100 \\
\hline \multirow{4}{*}{ F6 } & $\begin{array}{l}\text { SPF } \\
\text { FPS }\end{array}$ & $2.4 \pm 0.2$ & $\begin{array}{c}2.3 \pm \\
0.3\end{array}$ & $\begin{array}{c}2.4 \pm \\
0.4\end{array}$ & $\begin{array}{c}2.3 \pm \\
0.2\end{array}$ & $\begin{array}{c}2.2 \pm \\
0.3\end{array}$ & $\begin{array}{c}2.0 \pm \\
0.3 \\
\end{array}$ & $\begin{array}{c}2.3 \pm \\
0.4\end{array}$ & $\begin{array}{c}2.0 \pm \\
0.2\end{array}$ & $2.3 \pm 0.2$ & $\begin{array}{c}2.4 \pm \\
0.2 \\
\end{array}$ & $\begin{array}{c}2.2 \pm \\
0.2\end{array}$ & $\begin{array}{c}2.4 \pm \\
0.2\end{array}$ \\
\hline & $\lambda_{c}$ & 390 & 390 & 390 & 390 & 390 & 390 & 390 & 390 & 390 & 390 & 390 & 390 \\
\hline & $\begin{array}{c}\text { pH value } \\
\text { Valor de } \mathrm{pH}\end{array}$ & 4.69 & 4.77 & 4.79 & 4.84 & 4.61 & 4.65 & 4.72 & 4.84 & 4.41 & 4.56 & 4.61 & 4.73 \\
\hline & $\begin{array}{l}\text { Apparent } \\
\text { viscosity } \\
\text { Viscosidade } \\
\text { aparente }\end{array}$ & 21500 & 20100 & 24200 & 25100 & 11500 & 11300 & 13500 & 6100 & 31000 & 27400 & $\begin{array}{c}2750 \\
0\end{array}$ & 26200 \\
\hline
\end{tabular}

Legend: SPF: Sun Protection Factor (media \pm desvio padrão) $\lambda \mathrm{c}$ : Critical wavelenght

Legenda: FPS: Fator de Proteção Solar (average \pm standard deviation) $\lambda \mathrm{c}$ : Comprimento de onda crítico 
Base emulsion SPF was approximately 1.0. Analyzing the absorbance spectrum of the formulation F1 (Figure $1)$, values close to zero were verified along the whole ultraviolet spectrum (290 to $400 \mathrm{~nm})$, indicating an insignificant radiation absorption / reflection and, consequently, the absence of a photoprotective profile for the base emulsion.
O FPS da emulsão base foi, aproximadamente, 1.0. Ao avaliar o espectro da absorbância da formulação F1 (Figura 1), verificou-se valor próximo à zero ao longo do espectro ultravioleta (290 a $400 \mathrm{~nm}$ ), ou seja, não houve absorção/reflexão significativa da radiação e, consequentemente, a emulsão base não apresentou perfil fotoprotetor.

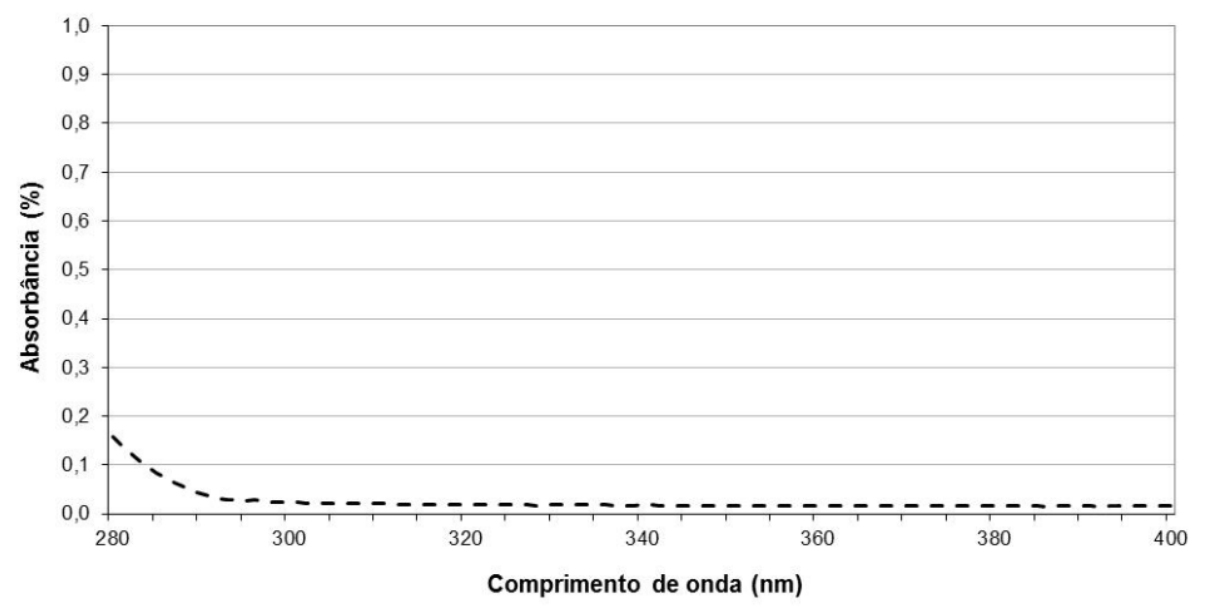

Figure 1 - Formulation F1 absorption profile (initial condition)

Figura 1 - Perfil de absorbância da formulação F1 (condição inicial)

Formulations containing titanium dioxide associated with zinc oxide (F3) and quercetin alone (F6) were stable during the stability test, according to data presented in Table 3. The emulsions presented a homogeneous aspect, without alterations in color or odor. In addition, the formulations did not present variation in the SPF, critical wavelength and $\mathrm{pH}$ values when compared to the initial condition, demonstrating physical and chemical stability. Apparent viscosity presented the same pattern obtained for the base emulsion(F1).

With reference to the absorbance spectrum of the formulation F3 (Figure 2), a satisfactory absorption profile in the UVA region (from 320 to $400 \mathrm{~nm}$ ) was observed, as well as a more effective absorption in the UVB region (from 280 to $320 \mathrm{~nm}$ ). Formulation F6 presented a good absorbance profile along the whole UV radiation spectrum (UVB and UVA), even in low concentrations of the bioactive compound (Figure 3). The critical wavelength values of this formulation indicate that quercetin is suitable for the development of formulations with broad spectrum protection ${ }^{[13]}$.
As preparações contendo dióxido de titânio associado ao óxido de zinco (F3) e quercetina isolada (F6) apresentaram-se estáveis ao longo do teste de estabilidade, conforme parâmetros apresentados na Tabela 3. O aspecto se manteve homogêneo, sem alterações de cor e odor. Além disso, as formulações não apresentaram variações dos valores médios de FPS, comprimento de onda crítico e $\mathrm{pH}$ em relação ao inicial, demonstrando estabilidade física e química. A viscosidade aparente apresentou perfil com o mesmo padrão de resposta da emulsão base (F1).

A partir do espectro de absorção da formulação F3 (Figura 2), observou-se que houve perfil de absorção satisfatório na região do UVA (de 320 a $400 \mathrm{~nm}$ ) e absorção mais efetiva na região do UVB (de 280 a 320 $\mathrm{nm}$ ). A formulação F6 apresentou bom perfil de absorção ao longo de todo o espectro de radiação UV (UVB e UVA), mesmo em baixa concentração do composto bioativo (Figura 3). Os valores de comprimento de onda crítico dessa formulação indicam que a quercetina é adequada para o desenvolvimento de formulações com amplo espectro de proteção ${ }^{[13]}$. 


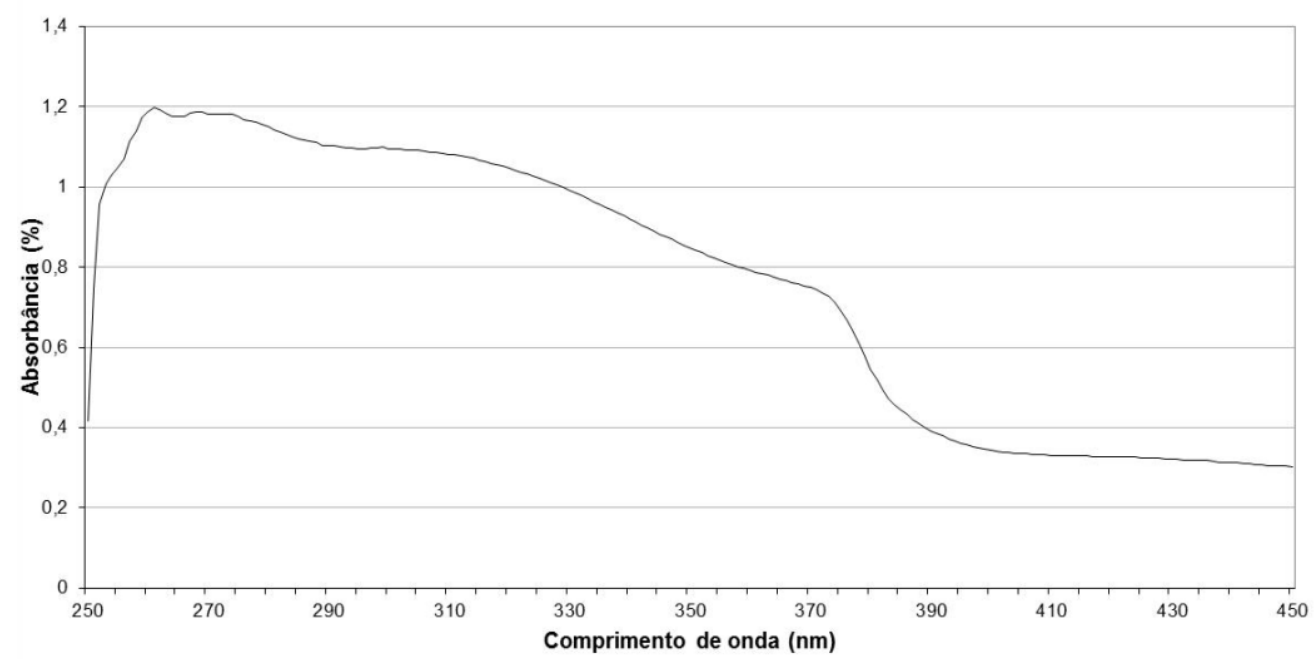

Figure 2 - Formulation F3 absorption profile (initial condition).

Figura 2 - Perfil de absorbância da formulação F3 (condição inicial).

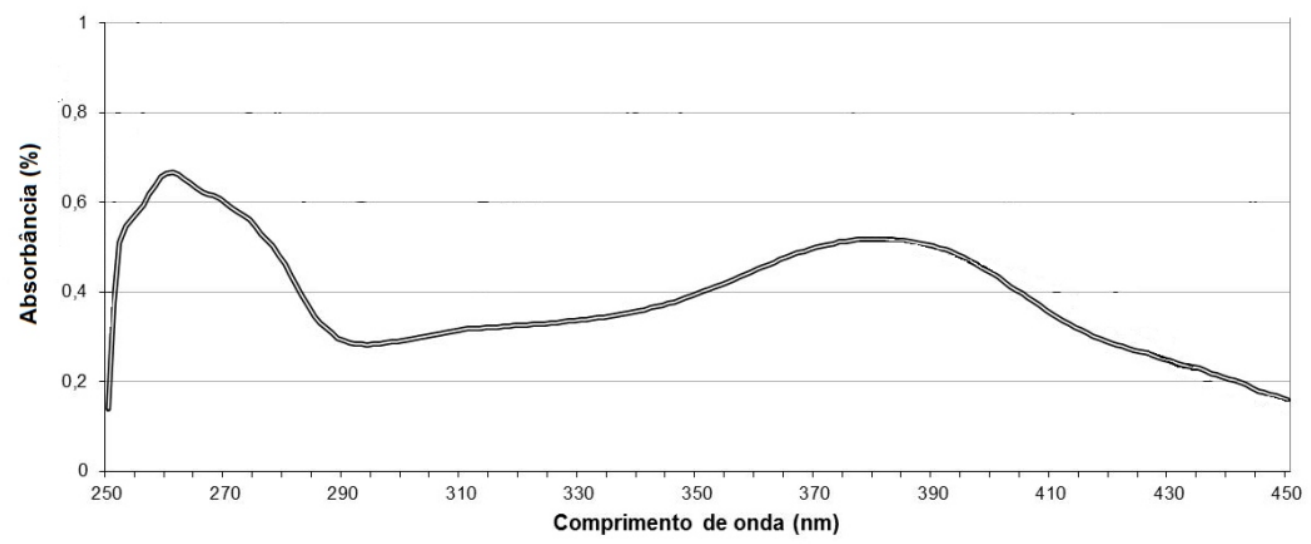

Figure 3 - Formulation F6 absorption profile (initial condition)

Figura 3 - Perfil de absorbância da formulação F6 (condição inicial)

Formulation F2, which contained an association of all the active compounds (quercetin, titanium dioxide and zinc oxide), developed an aspect considered inadequate. After preparation, the formulation presented an intense yellow color and during the stability test, an intense darkening was observed. This was perceptible, in all the storage conditions after the $30^{\text {th }}$ day of analysis..

The darkened aspect of the formulation $\mathrm{F} 2$ interfered in its functional characterization, for the film formation over the substrate was irregular and prevented the adequate passage of the UV radiation beam generated
A formulação $\mathrm{F} 2$, contendo associações de todos os compostos ativos (quercetina, dióxido de titânio e óxido de zinco), desenvolveu aspectos considerados inadequados. Após a manipulação, a formulação apresentou intensa coloração amarelada. Durante o período do teste de estabilidade, observou-se seu intenso escurecimento. Tal fato ocorreu, perceptivelmente, logo após o $30^{\circ}$ dia de análise, em todas as condições.

$\mathrm{O}$ aspecto escurecido da formulação $\mathrm{F} 2$ dificultou a caracterização funcional, pois a formação do filme sobre o substrato foi irregular, o que impediu a 
by the equipment. The samples were considered inadequate due to their modification in aspect and the in vitro photoprotective efficacy values were not registered. Figure 4 illustrates the formulation F2 absorbance profile in the initial condition and after 30 days in the high temperature storage. A modification in the general absorbance profile could be noticed. adequada passagem do feixe da radiação UV gerada pelo equipamento. Diante disso, em função da modificação do aspecto, as amostras foram consideradas inaptas, assim, não se registrando os valores dos parâmetros da eficácia fotoprotetora in vitro. A Figura 4 ilustra a absorbância da formulação F2 nas condições inicial e após 30 dias em temperatura elevada. Verificou-se que houve modificação do perfil geral de absorção.

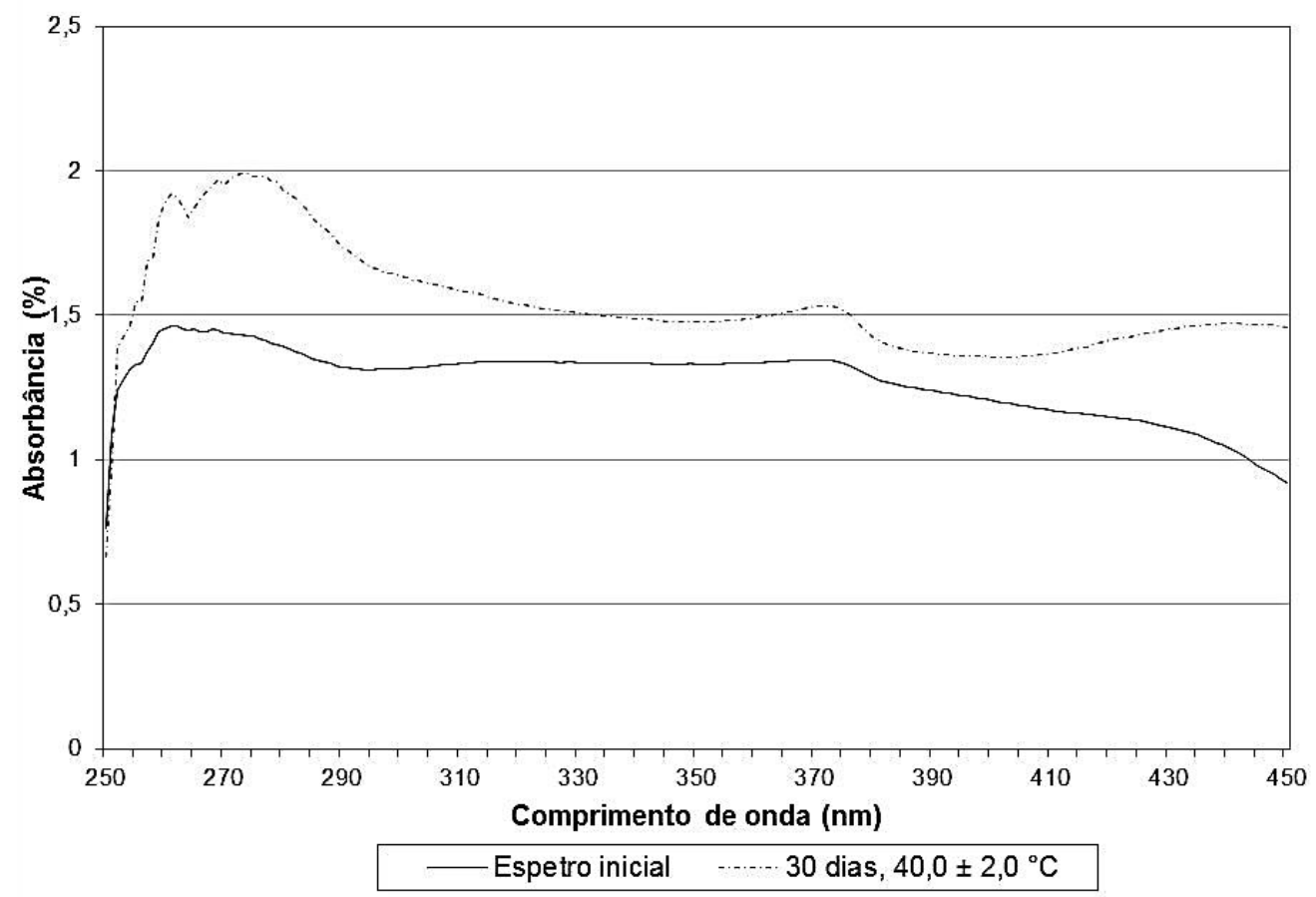

Figure 4 - Formulation F2 absorption profiles under initial condition and after 30 days in high temperature $\left(40.0 \pm 2.0^{\circ} \mathrm{C}\right)$

Figura 4 - Perfis de absorbância da formulação F2 nas condições inicial e após 30 dias em temperatura elevada $\left(40.0 \pm 2.0^{\circ} \mathrm{C}\right)$

For the formulations F4 (association of $2.5 \% \mathrm{w} / \mathrm{w}$ of quercetin and $5.0 \% \mathrm{w} / \mathrm{w}$ of titanium dioxide) and F5 (association of $2.5 \% \mathrm{w} / \mathrm{w}$ of quercetin and $5.0 \% \mathrm{w} / \mathrm{w}$ of zinc oxide) a slight color modification (darkening) for the high temperature storage condition was observed. In addition, the presence of the flavonoid could have formed agglomerates that hampered the spreading of the formulations over the PMMA substrate. Therefore, it was inferred that quercetin developed a characteristic that could not provide a good spreading profile of the formulations in the course of its shelf life. Considering the same criteria used for the formulation F2, the photoprotective efficacy values were not considered for the samples presenting aspect modifications.
Para as formulações F4 (associação de $2.5 \% \mathrm{p} / \mathrm{p}$ de quercetina e $5.0 \% \mathrm{p} / \mathrm{p}$ de dióxido de titânio) e F5 (associação de $2.5 \% \mathrm{p} / \mathrm{p}$ de quercetina e $5.0 \% \mathrm{p} / \mathrm{p}$ de óxido de zinco) foi possível observar leve modificação da coloração (escurecimento) na condição de temperatura elevada. Ainda, a presença do flavonoide pode ter formado aglomerados que dificultaram o espalhamento das formulações sobre o substrato de PMMA. Infere-se, segundo o exposto, que a quercetina desenvolveu característica que dificultou o perfil de espalhamento das preparações durante o envelhecimento. Considerando o mesmo critério utilizado para a formulação F2, os valores de eficácia fotoprotetora não foram considerados para as amostras que apresentaram modificação no aspecto. 


\section{Discussion}

The results obtained for formulations F1, F3 and F6 showed that the method used for the photoprotective efficacy determination was satisfactory, since it provided reproducible data. Limitations identified during the study were due to aspect alterations of some samples, a fact that did not compromise the methodology applied in the photoprotective efficacy estimation.

Metallic ions, such as titanium and zinc, can promote the flavonoids' oxidation through the formation of oxygen reactive species, while the darkening can occur due to oxidation, complexation reactions and/or metalpolyfenol interactions. Considering the results and experimental conditions, it can be suggested that formulations containing quercetin associated with the inorganic filters titanium dioxide and zinc oxide, and in the proportions employed, presented instability, or even incompatibility, demonstrated only in the stability evaluation. It is also important to mention that the stability profile can also be compromised by the vehicle (raw materials and its quantitative proportions), storage condition and packaging material, among other factors.

The active compounds studied (titanium dioxide, zinc oxide and quercetin) are safe for use in sunscreen formulations, since chemical reactions or other interactions did not alter the original characteristics. Quercetin complexation and oxidation reactions promote an increase in absorbance, of approximately $420 \mathrm{~nm}$ (visible radiation), corroborating the spectral profiles and color modification of the products ${ }^{[15]}$.

Velasco and co-workers (2008) had studied the association of flavonoids with inorganic UV filters, and obtained satisfactory synergic results without noticing signs of incompatibility between the compounds throughout the study. However, the flavonoid concentration, rutin, was only $0.1 \% \mathrm{w} / \mathrm{w}$ and the compound was dissolved on the emulsified system [13]. Choquenet and co-workers (2008) studied the photoprotective efficacy of rutin and quercetin in high concentrations, associated with the filters titanium dioxide and zinc oxide, and also obtained formulations with broad spectrum and high values of SPF. However, stability tests as a function of time were not mentioned in order to evaluate the existence of possible interactions between the compounds that could provoke modifications similar to the ones observed for this study [16].

\section{Discussão}

A avaliação dos resultados para as formulações F1, F3 e F6 providenciou evidências de que o método empregado para a determinação da eficácia fotoprotetora foi satisfatório, pois forneceu dados considerados reprodutíveis. Portanto, as limitações identificadas no estudo se relacionaram com as amostras nas quais houveram modificações de aspecto e se deveram, exclusivamente, à natureza dessas, não comprometendo a metodologia aplicada na estimativa da eficácia fotoprotetora.

Íons metálicos, como o titânio e o zinco, podem promover a oxidação dos flavonoides pela formação de espécies reativas de oxigênio, enquanto o escurecimento pode ocorrer devido à oxidação, reações de complexação e/ou interações metalpolifenol. Em função dos resultados e condições experimentais, permite-se sugerir que as formulações contendo quercetina associada aos filtros dióxido de titânio e óxido de zinco, nas proporções empregadas, apresentaram instabilidade, ou mesmo incompatibilidade, somente constatada no decorrer da avaliação da estabilidade. Ressalta-se que tal perfil de estabilidade também pode ter sido comprometido pelo veículo (adjuvantes farmacotécnicos e suas proporções quantitativas), condição de armazenamento e material de acondicionamento, entre outros fatores.

Os compostos ativos utilizados (dióxido de titânio, óxido de zinco e quercetina) são seguros para uso em formulações cosméticas fotoprotetoras desde que não haja reações químicas ou interações de outra natureza que alterem suas características originais. As reações de complexação e oxidação da quercetina provocam aumento na absorbância em, aproximadamente, 420 $\mathrm{nm}$ (radiação visível), o que corroboraria com as modificações da cor dos produtos e de seus perfis espectrais ${ }^{[15]}$.

Velasco e colaboradores (2008) haviam estudado a associação de flavonoides com filtros solares inorgânicos, obtendo resultados sinérgicos satisfatórios e não tendo observado, ao longo do estudo, sinais de incompatibilidade entre estes filtros. No entanto, a concentração do flavonoide, rutina, foi de apenas $0.1 \% \mathrm{p} / \mathrm{p}$ e esta estava dissolvida no sistema emulsionado ${ }^{[13]}$. Choquenet e colaboradores (2008) estudaram a eficácia fotoprotetora da rutina e quercetina em concentrações elevadas, associadas aos filtros dióxido de titânio e óxido de zinco, também obtendo formulações com amplo espectro e elevado valor de FPS. No entanto, testes de estabilidade em função do tempo não foram relatados para avaliar a existência de interações entre os compostos que pudessem provocar modificações semelhantes às observadas no presente estudo ${ }^{[16]}$. 


\section{Conclusion}

It was shown that the association of quercetin with inorganic filters traditionally used in the Cosmetic Industry (titanium dioxide and zinc oxide) was not suitable due to the negative interactions that occurred between the bioactive compound and the metals, only identified during the course of the stability study. This highlights the importance of monitoring the functional stability of photoprotective formulations. However, considering the in vitro estimated photoprotective efficacy of the isolated flavonoid, its use can still be explored as an alternative substitute to classic UV filters, in addition to its antioxidant properties.

\section{Acknowledgements}

FAPESP and CNPq.

\section{Conclusão}

Constatou-se que não foi adequado utilizar a quercetina associada aos filtros solares físicos tradicionalmente empregados pela Indústria Cosmética (dióxido de titânio e óxido de zinco) devido às interações negativas que ocorreram entre o composto bioativo e os metais, somente identificadas ao longo do tempo de realização do teste de estabilidade, ressaltando a relevância do acompanhamento da estabilidade funcional de formulações fotoprotetoras. No entanto, em função da eficácia fotoprotetora estimada in vitro apresentada pelo flavonoide isolado, seu uso ainda pode ser explorado como substituto alternativo aos filtros solares clássicos, somado às suas propriedades antioxidantes.

\section{Agradecimentos}

FAPESP - Fundação de Amparo à Pesquisa do Estado de São Paulo

\section{Conflito de interesses}

Os autores declaram que não existem relações financeiras ou pessoais que puderam ser vistas como um potencial conflito de interesses.
The authors declare that there are no financial or
personal relations that could be viewed as a potential conflict of interests.

\section{References / Referências}

[1]. Svobodova A, Walterova D, Vostalova J. Ultraviolet light induced alteration to the skin. Biomed Pap Med Fac Univ Palacky Olomuc Czech Repub 2006; 25:38-1.

[2]. Palm MD; O’Donoghue MN. Update on photoprotection. Dermatol Ther 2007; 20:360-376.

[3]. Shaath NA. Evolution of modern sunscreen chemicals. In: Lowe, N.J.; Shaath, M.A.; Pathak, M.A. Sunscreens: development, evaluation, and regulatory aspects; 1997.p. 3-33.

[4] Giokas DL, Salvador A, Chisvert A. UV filters: from sunscreen to human body and the environment. Trac-Trend Anal Chem 2007; 26: 360-374.

[5]. Flor J, Davolos MR, Corrêa MA. Protetores solares. Quim Nova 2007; 30:153-158.

[6]. Schlumpf M, Schmid P, Durrer S, Conscience M, Maerkel K, Henseler M, Gruetter M, Herzog I, Reolon S, Ceccatelli R, Faass O, Stutz E, Jarry H, Wuttke W, Lichtensteiger W. Endocrine activity and developmental toxicity cosmetic UV filters - an update. Toxicology 2004; 205: 113-122.
[7]. Maier T, Korting HC. Sunscreens - which and what for? Skin Pharmacol Physiol 2005; 18: 253-262. [8]. European Pharmacopoeia. EP 6thed. Strasbourg: Council of Europe, 2007.

[9]. United States Pharmacopeia: USP34; The National Formulary: NF29. Rockville: United States Pharmacopeial Convention, 2011; xvi:xviii-2.

[10]. Diffey BL. Indices of protection from in vitro assay of sunscreens. In: Lowe, NJ, Shaath, MA, Pathak MA. Sunscreens: development, evaluation, and regulatory aspects. 2.ed. New York: Marcel Dekker; 1997. p. 589-600.

[11]. Springsteen A, Yurek R, Frazier M, Carr KF. In vitro measurement of sunprotection factor of sunscreens by diffuse transmittance. Anal Chim Acta 1999; 380: 155164.

[12]. Velasco, MVR, Sarruf FD, Oliveira CA, Silva APM, Consiglieri VO, Kaneko TM, Baby AR. Influence of a bioactive substance on the physicochemical and functional stability of sunscreen emulsions. Biomed \& Biopharm Research 2012; 9:119-130.
[13]. Velasco MVR, Sarruf FD, Salgado-Santos IMN, Haroutiounian Filho CA, Kaneko TM, Baby AR. Broad spectrum bioactive sunscreens. Int $\mathrm{J}$ Pharm 2008; 363: 50-57.

[14]. Brasil. Agência Nacional de Vigilância Sanitária. Guia de estabilidade de produtos cosméticos. Brasília, 2004; 45p.

[15]. Makris DP, Rossiter JT. Heat-induced, metalcatalyzed oxidative degradation of Quercetin and Rutin (Quercetin 3-O-Rhamnosylglocoside) in aqueous model system. J Agr Food Chem 2000; 48: 3830-3838.

[16]. Choquenet B, Couteau C, Paparis E, Coiffard LJM. Quercetin and rutin as potential sunscreen agents: determination of efficacy by an in vitro method. J Nat Products 2008; 71: 1117-1118. 